\title{
Effet de l'extrusion de la féverole (Vicia faba) sur les flux intestinaux d'azote et d'amidon chez la vache laitière en production
}

\author{
C Benchaar, M Vernay *, C Bayourthe, R Moncoulon
}

\author{
ENSAT, laboratoire d'ingénierie agronomique, 145 avenue de Muret, \\ 31076 Toulouse Cedex, France
}

(Reçu le 18 novembre 1991; accepté le 22 avril 1992)

\begin{abstract}
Résumé - Quatre vaches Holstein en lactation, pourvues de canules (rumen; duodénum; iléon) ont été utilisées afin d'étudier l'effet de l'extrusion à $195^{\circ} \mathrm{C}$ de la féverole (Vicia faba cv Castel) sur la digestion de la matière organique (MO), de l'azote $(\mathrm{N})$ et de l'amidon. Le Cr-EDTA, $\mathrm{YbCl}_{3}$ et les bases puriques ont été choisis comme marqueurs respectifs des phases liquide et solide et des bactéries. La substitution de la féverole crue par la féverole extrudée dans la ration de base (ensilage de maïs plante entière, foin de ray-grass d'italie, maïs grain broyé) n'a affecté ni les paramètres fermentaires, ni la digestion ruminale apparente de la $\mathrm{MO}(39 \%)$; la digestion apparente de l'amidon a été accrue (72 vs $58 \%$ ), alors que celle de $\mathrm{N}$ alimentaire a été diminuée (45 vs $52 \%$ ). L'efficacité de la synthèse bactérienne ( $\mathrm{gN} / \mathrm{kg}$ de MORDR) a été augmentée (25 vs 22). Les flux duodénaux de $\mathrm{N}$ non ammoniacal, $N$ bactérien et $N$ alimentaire ont été plus importants, soit respectivement $(\mathrm{g} / \mathrm{j}): 409$ vs 366, 216 vs 194 et 193 vs 172. Parallèlement, la digestion intestinale apparente (g/j) de ces 3 formes azotées a été plus élevée : 268 vs 229 ( $\mathrm{N}$ non ammoniacal), 181 vs 161 ( $\mathrm{N}$ bactérien) et 87 vs 69 ( $\mathrm{N}$ alimentaire); celle de l'amidon étant plus faible (975 vs 1612 ) et celle de la MO demeurant inchangée, 4243 environ. Pour la féverole crue, les valeurs PDIA, PDIN et PDIE ( $\mathrm{g} / \mathrm{kg}$ de MS) sont : 33,175 , et 127; les valeurs correspondantes pour la graine extrudée étant : 58,147 et 178 .
\end{abstract}

féverole / extrusion / protéine / amidon / flux intestinal

Summary - Incidence of bean (Vicia faba) extrusion on starch and nitrogen intestinal flows in lactating cows. Four mature lactating Holstein cows fitted with permanent ruminal, duodenal and ileal cannulae were used to study the effect of extrusion at $195^{\circ} \mathrm{C}$ of beans (Nicia faba $\mathrm{cv}$ Castel) on organic matter (OM), nitrogen $(N)$ and starch degradation in the rumen and their flow to and absorption from the small intestine. The test protein sources, raw beans (RB) and extruded beans (EB), provided about $45 \%$ of the dietary protein. The diets were composed of $23.1 \%$ beans, $56.2 \%$ corn silage, $10.1 \%$ corn grain and $10.7 \%$ Italian rye-grass hay on a DM basis; the diets were isocaloric (4.5 $\mathrm{Mcal} / \mathrm{kg}$ of DM) and isonitrogenous (14\% of DM). Cr-EDTA, $\mathrm{YbCl}_{3}$ and purines were used as liquid, particulate and bacterial markers respectively. Extruding the beans did not influence intraruminal $\mathrm{pH}$ (6.6), ammonia- $\mathrm{N}(99 \mathrm{mgl})$ and volatile fatty acids (97 mM/l) concentrations. Apparent digestibility in total tract of energy, OM, N and starch were not affected with inclusion of EB instead of RB, the corresponding mean values were: $66,68,64$ and $95 \%$ of intake. Apparent ruminal digestion of OM,

* Correspondance et tirés à part 
starch and $N$ in the forestomach were 39,58 and $52 \%$ for diets containing $R B$ and 38,72 and $45 \%$ for $E B$ diets; efficiency of bacterial protein synthesis ( $g \mathrm{~N} / \mathrm{kg}$ organic matter truly digested in the stomach) was higher for $E N$ diets compared with RN diets (25 vs 22). Eating diets including $E B$ increased nonammonia- $N$, bacterial- $N$ and dietary- $N$ flows ( $g / d$ ) to the duodenum compared with diets containing RB: 409 vs 366, 216 vs 194 and 193 vs 172 respectively; while starch flow was reduced (1.5 vs 2.2 $\mathrm{kg} / \mathrm{d})$ and $O M$ flow was unchanged $(9.8 \mathrm{~kg} / \mathrm{d})$. Apparent digestion from the small intestine $(\mathrm{g} / \mathrm{d})$ of nonammonia- $N$, bacterial $N$ and dietary- $N$ were higher for $E B$ diets: 268 vs 229,181 vs 160 and 87 vs 69 respectively; meanwhile, starch digestion decreased (975 vs 1612). The PDIA, PDIN and PDIE contents ( $\mathrm{g} / \mathrm{kg}$ of $D M$ ) of the $R B$ were 33, 175 and 127 respectively; the corresponding values after extrusion were: 58,147 and 178 .

\section{bean / extrusion / protein / starch / intestinal flow}

\section{INTRODUCTION}

L'intérêt nutritionnel de la féverole (Vicia faba) dans l'alimentation animale réside dans sa composition chimique (CerningBeroard et Filiatre, 1977); tout comme les céréales d'une part et les tourteaux d'autre part, elle est riche en amidon ( $40 \%$ de MS) et en matières azotées ( $32 \%$ de MS). Cependant, ce protéagineux est caractérisé in vitro, par une faible digestibilité de l'amidon (Cerning-Beroard, 1977) et une forte solubilité de l'azote (Michalet-Doreau et al, 1987) et in situ, par une dégradabilité de l'azote ( $N$ ) élevée (Michalet-Doreau et al, 1987; Cros et al, 1991). En conséquence, la féverole ne pourrait être substituée aux tourteaux et graines de soja dans l'alimentation des ruminants qu'après traitement technologique. Au cours d'une expérimentation préliminaire réalisée in situ, sur vaches taries (Benchaar et al, 1992), nous avons mis en évidence que la cuissonextrusion de la féverole à $195^{\circ} \mathrm{C}$ insolubilise ses protéines ( 37 vs $56 \% \mathrm{Nt}$ ), réduit leur dégradabilité ruminale $(66,5$ vs $73,3 \%$ $\mathrm{N}$ initial) et augmente la part de $\mathrm{N}$ alimentaire qui disparaît au cours du tran-sit dans les régions postruminales (29 vs $22 \% \mathrm{Nt}$ initial). Par contre, McMeniman et Armstrong (1979) notent que l'extrusion (105 $\left.{ }^{\circ} \mathrm{C}\right)$ de cette graine ne modifie pas les flux duodénaux de $\mathrm{N}$ total, $\mathrm{N}$ microbien et $\mathrm{N}$ alimentaire chez la vache. Signalons encore que les traitements hydrothermiques, qui entraînent une gélatinisation des granules d'amidon et un passage en solution d'une fraction de l'amylose, facilitent l'amylolyse in vitro de la féverole (Cerning-Beroard, 1977). L'extrusion pourrait donc accélérer la digestion ruminale de l'amidon et, par voie de conséquence, accroître la synthèse des protéines microbiennes.

L'objectif de cette étude est de confirmer l'hypothèse d'un effet protecteur de l'extrusion à $195^{\circ} \mathrm{C}$ sur les matières azotées (MA) de la féverole (Vicia faba cv Castel) et de déterminer l'incidence de ce traitement sur la digestion de l'amidon chez la vache laitière. Ces effets sont évalués au niveau des flux intestinaux : duodénal, iléal et fécal.

\section{MATÉRIEL ET MÉTHODES}

\section{Animaux}

Quatre vaches Holstein en lactation (production laitière moyenne : $35 \pm 3,6 \mathrm{~kg} / \mathrm{j}$ ) d'un poids moyen de $650 \mathrm{~kg}$ et munies de canules (rumen; duodénum proximal; iléon terminal) ont été utilisées pour ce travail. 


\section{Régimes}

Au cours de 2 expérimentations, espacées de 3 semaines, les animaux ont reçu en 4 repas égaux $(6,12,18$ et $24 \mathrm{~h})$ une ration ( $\mathrm{kg}$ de MS. $\mathrm{j}^{-1}$ par animal) composée d'ensilage de maïs plante entière (10), de foin de ray-grass d'Italie $(1,9)$ et de maïs grain broyé $(1,8)$. De plus, chaque vache a reçu $4 \mathrm{~kg}$ de graines de féverole broyées : crues ou extrudées* à $195^{\circ} \mathrm{C}$, et $50 \mathrm{~g}$ d'un complément minéral et vitaminique. Les rations ont été formulées de telle sorte que $45 \%$ des MA soient apportées par la féverole. La composition des différents aliments est rapportée dans le tableau $\mathrm{l}$.

\section{Procole experimental et traitement des échantillons}

Le Cr-EDTA (Binnerts et al, 1968) et $\mathrm{YbCl}_{3}$ (Ellis et al, 1982) ont été utilisés comme marqueurs respectifs des phases liquide et solide; les protéines bactériennes étant identifiées par le biais des bases puriques. Après $15 \mathrm{j}\left(\mathrm{j}_{1}-\mathrm{j}_{15}\right)$ d'adaptation à la ration, chaque vache a reçu, via la canule ruminale, une même dose de Cr-EDTA $(1,4 \mathrm{~g} \mathrm{Cr})$ et de $\mathrm{YbCl}_{3}(2,0 \mathrm{~g} \mathrm{Yb})$. Au cours des $7 \mathrm{j}$ suivants $\left(\mathrm{j}_{16}-\mathrm{j}_{22}\right)$, les solutions de marqueurs $(\mathrm{g} / \mathrm{j})$ ont été infusées en continu dans le rumen à raison de :2,8 de $\mathrm{Cr}$ et 2,0 de $\mathrm{Yb}$. Les prélève- ments de digesta (ruminal; duodénal; iléal) ont été réalisés entre $j_{20}$ et $j_{24}$. Dans un premier temps, pour estimer les flux digestifs, du contenu duodénal $(250 \mathrm{ml})$ et iléal $(100 \mathrm{ml})$ a été prélevé pendant $3 \mathrm{j}$ consécutifs $\left(\mathrm{j}_{20}-\mathrm{j}_{22}\right)$ à raison de 4 prises/j. Après homogénéisation, les digesta duodénaux ont été fractionnés selon la méthode de double marquage (Faichney, 1980). Dans un deuxième temps $\left(\mathrm{j}_{23}-\mathrm{j}_{24}\right)$ du jus ruminal a été prélevé (2L; $15 \mathrm{~min}$ avant, puis 1,2 et $4 \mathrm{~h}$ après les repas de 6 et $18 \mathrm{~h}$ ) afin de mesurer les paramètres fermentaires. Après mesure du $\mathrm{pH}$ le liquide ruminal a été filtré à travers un tamis de $1 \mathrm{~mm}$ de maille. Le filtrat obtenu a été fractionné en vue des dosages d'ammoniac $\left(\mathrm{N}-\mathrm{NH}_{3}\right)$ et d'acides gras volatils (AGV) d'une part et de l'isolement du culot bactérien d'autre part (Jouany et Thivend, 1972). Au fur et à mesure de leur obtention, les filtrats ruminaux, les fractions duodénales et le matériel iléal ont été congelés $\left(-20^{\circ} \mathrm{C}\right)$ jusqu'au moment des analyses. Les mesures des quantités de MS réellement ingérées, de même que la récolte totale des fèces ont été effectuées entre $j_{20}$ et $j_{24}$ afin d'évaluer la digestibilité des principaux constituants de la ration et le taux de récupération des marqueurs infusés.

\section{Analyses}

Les teneurs en MS, matière organique (MO) et MA des aliments, des refus, des fèces des di-

Tableau I. Matière sèche (\%o) et composition ( $\mathrm{g} / \mathrm{kg}$ de MS) des différents aliments de la ration.

\begin{tabular}{|c|c|c|c|c|c|c|c|}
\hline Aliments & MS & $M O$ & $M A$ & NDF & $A D F$ & $L I$ & $A M$ \\
\hline Ensilage de maïs & 334,0 & 948,6 & 98,1 & 521,7 & 234,8 & 46,4 & 294,0 \\
\hline Foin de ray-grass & 922,2 & 928,1 & 59,6 & 632,5 & 372,6 & 91,4 & 21,0 \\
\hline Maïs & 883,8 & 985,9 & 84,4 & 122,0 & 24,7 & 6,8 & 684,0 \\
\hline Féverole: crue & 883,6 & 956,5 & 256,6 & 202,9 & 103,8 & 23,0 & 315,4 \\
\hline extrudée & 942,9 & 958,4 & 253,2 & 192,1 & 106,4 & 29,0 & 319,9 \\
\hline
\end{tabular}

Matières : sèche (MS), organique (MO) et azotée (MA); glucides membranaires obtenus à la suite du traitement par les détergents neutre (NDF) et acide (ADF de Van Soest); lignine (LI) et amidon (AM).

\footnotetext{
* Extrudeur bivis Werner et Pfleiderer (Continua 37)
} 
gesta et des bactéries ont été déterminées selon les techniques décrites dans le Journal Officiel des Communautés Européennes (1971); l'amidon étant estimé selon la méthode de l'amyloglucosidase commercialisée par Boehringer * La fraction $N$ soluble des protéines de la féverole a été évaluée en solution tampon (Vérité et Demarquilly, 1978). Les glucides membranaires des aliments, ont été dosés selon la méthode préconisée par Van Soest (1963) et Van Soest et Wine (1967, 1968). La teneur en $\mathrm{N}-\mathrm{NH}_{3}$ dans les digesta a été mesurée selon la procédure de Verdouw et al (1977); l'analyse des AGV a été réalisée par chromatographie en phase gazeuse selon la technique décrite par Jouany (1982). Les teneurs en bases puriques des bactéries, ruminales et intestinales, et des digesta intestinaux lyophilisés ont été évaluées selon les procédés de Ushida et al (1985). À partir des échantillons digestifs secs, le $\mathrm{Cr}$ a été extrait selon la technique de Siddons et al (1985); l'extraction de $\mathrm{Yb}$ a été réalisée d'après la méthode d'Ellis et al (1982). Ces 2 éléments ont été dosés par spectrophotométrie d'absorption atomique à l'aide d'une flamme air-acétylène pour le premier et d'un mélange protoxyde d'azoteacétylène pour le second.

\section{Calculs}

Les flux intestinaux des différents constituants de la ration, corrigés par les taux de récupération des marqueurs dans les fèces, ont été calculés par double marquage (Faichney, 1980) pour le duodénum (matériel hétérogène) et par simple marquage pour l'iléon. Les proportions de $\mathbf{N}$ bactérien dans $\mathrm{Nt}$ intestinal ont été estimées à partir du rapport : (marqueur bactérien/ $\mathrm{Nt}$ intestinal) / (marqueur bactérien/Nt bactérien ruminal). Les quantités de $\mathrm{MO}$ réellement dégradées dans le rumen (MORDR) ont été calculées en faisant la différence entre la MO d'origine bactérienne et la MO du flux duodénal.

L'exploitation statistique des résultats a été effectuée à l'aide du test $t$ de Student pour séries appariées. Les différences sont considérées comme significatives lorsque $P<0,05$.

\section{RÉSULTATS}

\section{Rations}

Les régimes offerts aux vaches au cours de l'expérimentation sont iso-azotés (14,0\% de MA), mais ils diffèrent par leurs teneurs en $\mathrm{N}$ soluble. En effet, après extrusion de la féverole à $195^{\circ} \mathrm{C}$, la fraction $\mathrm{N}$ soluble est significativement diminuée (36,5 vs $55,5 \% \mathrm{Nt}$ ). La substitution de la graine crue par la graine extrudée dans l'alimentation des animaux ne modifie pas l'ingestion de MS d'une part $(16,8 \mathrm{~kg} / \mathrm{j})$ et la digestibilité des principaux constituants de la ration d'autre part (tableaux III, IV et V).

\section{Paramètres fermentaires}

Le $\mathrm{pH}$ et les teneurs en $\mathrm{N}-\mathrm{NH}_{3}$ et AGVt du jus de rumen ne sont pas différentes pour les 2 rations (tableau II). Les valeurs moyennes étant de $6,6,99 \mathrm{mg} / \mathrm{l}$ et $97 \mathrm{mM} / \mathrm{l}$ respectivement; par ailleurs, la répartition qualitative des principaux AGV ne varie pas.

\section{Flux et digestion de la matière organique et de l'amidon}

Le remplacement de la féverole crue par la féverole extrudée dans le régime n'a pas d'incidence significative sur les flux intestinaux de la MO (tableau III).

De l'examen du tableau IV, il ressort que lorsque les vaches ingèrent la ration complémentée par la graine extrudée, la digestion ruminale apparente de l'amidon

\footnotetext{
* Boehringer, Mannheim; Réf 207748
} 
Tableau II. Influence de la complémentation de la ration par la féverole crue ( $\mathrm{FC})$ ou extrudée (FE) à $195^{\circ} \mathrm{C}$ sur les paramètres fermentaires ruminaux chez les vaches en lactation.

\begin{tabular}{lrr}
\hline $\begin{array}{l}\text { Parametres } \\
\text { fermentaires }\end{array}$ & \multicolumn{1}{c}{$F C$} & $F E$ \\
& & \\
\hline $\mathrm{pH}$ & $6,6^{\mathrm{a}} \pm 0,1$ & $6,5^{\mathrm{a}} \pm 0,04$ \\
& & \\
$\mathrm{~N}-\mathrm{NH}_{3}$ (mg/l) & $105,9^{\mathrm{a}} \pm 11,2$ & $91,3^{\mathrm{a}} \pm 5,6$ \\
$\mathrm{AGVt}(\mathrm{mM} /)$ & $97,4^{\mathrm{a}} \pm 0,2$ & $96,1^{\mathrm{a}} \pm 5,2$ \\
& & \\
AGV (\% AGVt) & & \\
Acétate & $63,7^{\mathrm{a}} \pm 0,9$ & $64,7^{\mathrm{a}} \pm 0,4$ \\
Propionate & $19,1^{\mathrm{a}} \pm 1,3$ & $19,8^{\mathrm{a}} \pm 0,6$ \\
Isobutyrate & $0,9^{\mathrm{a}} \pm 0,1$ & $0,7^{\mathrm{a}} \pm 0,1$ \\
Butyrate & $12,6^{\mathrm{a}} \pm 0,2$ & $12,3^{\mathrm{a}} \pm 0,2$ \\
Isovalérate & $2,1^{\mathrm{a}} \pm 0,2$ & $1,9^{\mathrm{a}} \pm 0,3$ \\
Valérate & $1,5^{\mathrm{a}} \pm 0,3$ & $1,7^{\mathrm{a}} \pm 0,1$ \\
\hline
\end{tabular}

Moyennes \pm ecart type; azote ammoniacal $\left(\mathrm{N}-\mathrm{NH}_{3}\right)$ et acides gras volatils totaux (AGVt). Les valeurs moyennes sur une même ligne affectées d'un même exposant ne sont pas statistiquement différentes $(P>0,05)$. est augmentée de $23,5 \%$; le flux duodénal baisse de $31,8 \%$ et parallèlement, la digestion dans l'intestin grêle (\% ingéré) est réduite de $40,6 \%$.

\section{Flux et digestion de la matière azotée}

Les flux intestinaux des différentes formes azotées : $\mathrm{N}$ non ammoniacal, $\mathrm{N}$ bactérien et $\mathrm{N}$ alimentaire, sont rapportés dans le tableau V. Quelle que soit la ration offerte aux animaux, le flux duodénal de $\mathrm{Nt}$ est supérieur à $\mathrm{N}$ ingéré; l'enrichissement étant plus conséquent après ingestion du régime contenant la graine traitée thermiquement à la place de la graine non traitée $(21,3$ vs $5,8 \%)$. Dans ce cas, les flux de $N$ non ammoniacal, $\mathrm{N}$ bactérien et $\mathrm{N}$ alimentaire à la sortie du rumen sont augmentés de $11,8 \%$ environ. La fraction de $\mathrm{N}$ alimentaire apparemment dégradée dans ce compartiment est réduite de $13,5 \%$, tandis

Tableau III. Ingestion $(\mathrm{kg} / \mathrm{j})$, flux $(\mathrm{kg} / \mathrm{j})$ et digestion de la matière organique dans le tube digestif de vaches en lactation recevant une ration complémentée par la féverole crue (FC) ou extrudée (FE) à $195^{\circ} \mathrm{C}$.

\begin{tabular}{|c|c|c|}
\hline Matière organique & $F C$ & $F E$ \\
\hline Ingestion & $16,1^{a} \pm 0,5$ & $15,8^{\mathrm{a}} \pm 0,2$ \\
\hline \multicolumn{3}{|l|}{ Flux } \\
\hline Duodénal & $9,9^{a} \pm 0,1$ & $9,7^{a} \pm 0,6$ \\
\hline lléal & $5,6^{a} \pm 0,1$ & $5,6^{a} \pm 0,2$ \\
\hline Fécal & $5,3^{a} \pm 0,2$ & $5,0^{\mathrm{b}} \pm 0,2$ \\
\hline \multicolumn{3}{|l|}{ Digestion ruminale } \\
\hline Apparente (\% ingéré) & $38,8^{a} \pm 0,1$ & $38,4^{a} \pm 3,8$ \\
\hline Réelle $(\mathrm{kg} / \mathrm{j})$ & $9,0^{a} \pm 0,1$ & $8,6^{a} \pm 0,5$ \\
\hline (\% ingéré) & $56,0^{a} \pm 0,2$ & $54,4^{a} \pm 3,5$ \\
\hline \multicolumn{3}{|l|}{ Digestion intestinale } \\
\hline$(\mathrm{kg} / \mathrm{j})$ & $4,3^{a} \pm 0,1$ & $4,2^{a} \pm 0,4$ \\
\hline (\% ingéré) & $26,8^{a} \pm 0,5$ & $26,4^{a} \pm 2,6$ \\
\hline Digestibilité totale (\% ingéré) & $67,0^{a} \pm 1,3$ & $68,8^{a} \pm 1,2$ \\
\hline
\end{tabular}

Moyenne \pm écart type; les valeurs moyennes sur une même ligne affectées d'un même exposant ne sont pas statistiquement différentes $(P>0,05)$. 
Tableau IV. Ingestion (kg/j), flux (kg/j) et digestion de l'amidon dans le tube digestif de vaches en lactation recevant une ration complémentée par de la féverole crue (FC) ou extrudée (FE) à $195^{\circ} \mathrm{C}$.

\begin{tabular}{|c|c|c|}
\hline Amidon & $F C$ & $F E$ \\
\hline $\begin{array}{l}\text { Ingestion } \\
\text { Flux }\end{array}$ & $5,2^{a} \pm 0,1$ & $5,3^{a} \pm 0,1$ \\
\hline Duodénal & $2,2^{a} \pm 0,2$ & $1,5^{b} \pm 0,1$ \\
\hline lléal & $0,6^{a} \pm 0,1$ & $0,5^{a} \pm 0,1$ \\
\hline Fécal & $0,3^{\mathrm{a}} \pm 0,01$ & $0,3^{a} \pm 0,01$ \\
\hline Digestion ruminale & & \\
\hline $\begin{array}{l}\text { apparente (\% ingéré) } \\
\text { Digestion intestinale }\end{array}$ & $57,9^{b} \pm 3,5$ & $71,5^{a} \pm 2,6$ \\
\hline$(\mathrm{kg} / \mathrm{j})$ & $1,6^{a} \pm 0,1$ & $0,9^{b} \pm 0,1$ \\
\hline (\% ingéré) & $31,0^{a} \pm 1,1$ & $18,4^{b} \pm 1,3$ \\
\hline (\% entrée) & $73,6^{a} \pm 1,5$ & $64,7^{b} \pm 1,9$ \\
\hline Digestibilité totale (\% ingéré) & $95,0^{a} \pm 1,6$ & $94,1^{a} \pm 1,2$ \\
\hline
\end{tabular}

Moyenne \pm écart type; les valeurs moyennes sur une même ligne affectées d'un même exposant ne sont pas statistiquement differentes $\{P>0,05)$.

que l'efficacité de la synthèse bactérienne (gN/kg de MORDR) est accrue de 17,7\% environ. Les flux iléaux des différentes formes azotées n'étant pas modifiés par le régime, la digestion intestinale apparente ( $\mathrm{g} / \mathrm{j}$ ) de $\mathrm{N}$ non ammoniacal, $\mathrm{N}$ bactérien et $\mathrm{N}$ alimentaire est respectivement augmentée de $17,0,12,7$ et $25,3 \%$ lorsque la féverole extrudée remplace la féverole crue. La digestion azotée dans les régions postiléales ne varie pas significativement au cours des 2 phases expérimentales.

\section{DISCUSSION}

L'extrusion de Vicia faba cv Castel entraîne une diminution de la fraction $\mathrm{N}$ soluble. Ce résultat est en accord, d'une part avec ceux de Michalet-Doreau et al (1987) qui montrent que pour Vicia faba cv Alto, la solubilité de $\mathrm{N}$ passe de 64,7 à $28,1 \%$ $\mathrm{Nt}$ après extrusion à $150^{\circ} \mathrm{C}$ et, d'autre part, avec ceux de Cros et al (1991) qui font apparaître une réduction de $\mathrm{N}$ soluble pour Vicia faba cv Talo extrudée à $120^{\circ} \mathrm{C}$ $(21,1$ vs $74,9 \% \mathrm{Nt})$. Si l'incidence du traitement thermique à $195^{\circ} \mathrm{C}$ est visible in vitro (insolubilisation de $\mathrm{N}$ ) et in situ, baisse de la dégradabilité de $N: 66,5$ vs $73,3 \%$ (Benchaar et al, 1992), il n'en est pas de même in vivo pour $\mathrm{N}-\mathrm{NH}_{3}$. En effet, l'extrusion de la féverole, en réduisant la fraction soluble et rapidement dégradable des protéines alimentaires, devrait affecter la production ruminale de $\mathrm{N}-\mathrm{NH}_{3}$ comme c'est le cas notamment pour le pois, le coton, le tourteau et les graines de soja (Thomas et al, 1979; Tagari et al, 1986; Focant et al, 1990). Or, la teneur en $\mathrm{N}^{-\mathrm{NH}_{3}}$ du jus de rumen n'est pas modifiée dans nos conditions expérimentales; des résultats similaires ont été obtenus par d'autres équipes pour les graines de féverole, de soja et de lupin (McMeniman et Armstrong, 1979; Van Dijk et al, 1983; Stern et al, 1985; MichaletDoreau et al, 1985; Benchaar et al, 1991). 
Tableau V. Ingestion $(g / j)$, flux $(g / j)$ et digestion des matières azotées dans le tube digestif de vaches en lactation recevant une ration complémentée par la féverole crue (FC) ou extrudée (FE) à $195^{\circ} \mathrm{C}$.

\begin{tabular}{|c|c|c|}
\hline Matières azotées & $F C$ & $F E$ \\
\hline Ingestion & $356,2^{\mathrm{a}} \pm 4,5$ & $347,9^{a} \pm 5,3$ \\
\hline \multicolumn{3}{|l|}{ Flux } \\
\hline Nt duodénal & $376,8^{b} \pm 0,1$ & $422,0^{a} \pm 2,3$ \\
\hline N non ammoniacal & $365,5^{b} \pm 1,7$ & $408,9^{a} \pm 1,1$ \\
\hline $\mathrm{N}$ bactérien & $194,2^{b} \pm 5,4$ & $216,2^{\mathrm{a}} \pm 8,4$ \\
\hline$N$ alimentaire ${ }^{*}$ & $172,1^{b} \pm 3,5$ & $192,6^{a} \pm 7,3$ \\
\hline Nt iléal & $142,1^{a} \pm 8,9$ & $145,7^{a} \pm 8,6$ \\
\hline $\mathrm{N}$ non ammoniacal & $136,7^{a} \pm 9,1$ & $141,2^{\mathrm{a}} \pm 7,8$ \\
\hline $\mathrm{N}$ bactérien & $33,8^{b} \pm 0,6$ & $35,4^{a} \pm 0,8$ \\
\hline $\mathrm{N}$ alimentaire & $102,8^{a} \pm 8,3$ & $105,8^{a} \pm 7,0$ \\
\hline Nt fécal & $124,9^{a} \pm 5,4$ & $125,7^{a} \pm 1,9$ \\
\hline \multicolumn{3}{|l|}{ Digestion ruminale } \\
\hline apparente (\% ingérée) & $51,7^{a} \pm 1,1$ & $44,7^{b} \pm 1,9$ \\
\hline \multicolumn{3}{|l|}{ Synthèse bactériennes (gN/kg) } \\
\hline MOADR & $31,0^{b} \pm 0,8$ & $35,8^{a} \pm 2,0$ \\
\hline MORDR & $21,5^{b} \pm 0,6$ & $25,3^{a} \pm 0,6$ \\
\hline \multicolumn{3}{|l|}{ Digestion intestinale } \\
\hline \multicolumn{3}{|l|}{$\mathrm{N}$ non ammoniacal } \\
\hline$(g / j)$ & $228,8^{b} \pm 8,2$ & $267,6^{a} \pm 6,7$ \\
\hline (\% ingéré) & $64,3^{\mathrm{b}} \pm 2,5$ & $76,9^{\mathrm{a}} \pm 1,7$ \\
\hline (\% entrée) & $62,7^{a} \pm 2,4$ & $65,5^{a} \pm 1,8$ \\
\hline \multicolumn{3}{|l|}{$\mathrm{N}$ bactérien } \\
\hline$(g / j)$ & $160,5^{b} \pm 4,9$ & $180,8^{a} \pm 7,5$ \\
\hline (\% entrée) & $82,6^{a} \pm 0,2$ & $83,6^{a} \pm 0,3$ \\
\hline \multicolumn{3}{|l|}{$\mathrm{N}$ alimentaire } \\
\hline$(g / j)$ & $69,3^{a} \pm 11,8$ & $86,8^{a} \pm 14,3$ \\
\hline (\% ingéré) & $19,5^{a} \pm 3,4$ & $25,0^{\mathrm{a}} \pm 4,0$ \\
\hline (\% entrée) & $40,2^{a} \pm 6,1$ & $44,9^{a} \pm 5,7$ \\
\hline Digestibilité totale (\% ingéré) & $95,0^{a} \pm 1,6$ & $94,1^{\mathrm{a}} \pm 1,2$ \\
\hline
\end{tabular}

Moyenne \pm écart type; azote total $(\mathrm{Nt}) ;{ }^{*}=\mathrm{N}$ alimentaire $+\mathrm{N}$ endogène; matière organique apparemment (MOADR) ou réellement (MORDR) dégradée dans le rumen. Les valeurs moyennes sur une même ligne affectées d'un même exposant ne sont pas statistiquement différentes $(P>0,05)$.

II est vrai que la concentration ruminale de $\mathrm{N}-\mathrm{NH}_{3}$ est un pool à plusieurs entrées et sorties. Elle ne traduit pas seulement une production à partir de $\mathrm{N}$ alimentaire, puisqu'elle dépend aussi de son utilisation par les microrganismes, du passage éventuel dans le sang et du recyclage par le biais des sécrétions salivaires. Dans nos essais, la teneur en $\mathrm{N}-\mathrm{NH}_{3}$ est toujours supérieure à $90 \mathrm{mg} / \mathrm{l}$; elle n'est donc pas limitante pour la croissance de la microflore (Madsen et Hvelplund, 1988). En fait, l'efficacité de la synthèse bactérienne exprimée en $\mathrm{gN} / \mathrm{kg}$ de MORDR augmente significativement, après extrusion de la féverole $(25,3$ vs 21,5); un effet identique étant signalé 
par Focant et al (1990) après traitement thermique du pois $(16,7$ vs 10,9$)$. Par contre, la synthèse microbienne n'est pas modifiée par la cuisson-extrusion (Stern et al, 1985; Pena et al, 1986; Benchaar et al, 1991) des graines de soja (42), coton (13) et lupin (22). Lorsque les animaux reçoivent la ration à base de féverole crue, la digestion ruminale de l'amidon par les $\alpha$ amylases bactériennes est en moyenne de $58 \%$. Pour les céréales, la fraction d'amidon qui est normalement dégradée dans le rumen est très variable et dépend de la structure du granule d'amidon; les valeurs s'échelonnent de 52 à $76 \%$ pour le sorgho, de 73 à $77 \%$ pour le maïs et le pois, de 92 à $95 \%$ pour l'orge (Waldo, 1973; Owens et al, 1986; Theurer, 1986; Focant et al, 1990). Après extrusion de la féverole, il apparaît que l'amylolyse dans les réservoirs de fermentation est plus intense; par voie de conséquence, la digestion postruminale de ce nutriment est fortement réduite. Par ailleurs, lors d'expérimentations réalisées in vitro, Cerning-Beroard (1977) a montré que l'utilisation digestive de l'amidon de la féverole est susceptible d'être profondément changée par l'extrusion. II s'agit surtout d'un accroissement de l'accessibilité du granule d'amidon à l' $\alpha$-amylase; la fraction facilement attaquable passant de 3 à $83 \%$ de MS. Une hausse de la digestion ruminale de l'amidon a également été observée, in vivo, par Theurer (1986) après traitement thermique des céréales (maïs, orge, sorgho). Par contre, Focant et al (1990) n'ont enregistré aucune variation significative de la fraction d'amidon digérée dans le rumen après extrusion du pois protéagineux (température d'extrusion non précisée).

Le flux duodénal de $\mathrm{Nt}$ est la résultante des différents flux : $\mathrm{N}$ ammoniacal, $\mathrm{N}$ bactérien et $\mathrm{N}$ alimentaire *, l'extrusion pou- vant avoir une incidence sur chacun d'eux. Dans nos conditions d'expérimentation, le flux $\mathrm{N}$ bactérien qui parvient au duodénum est augmenté lorsque, dans la ration, la graine extrudée remplace la graine crue (216 vs $194 \mathrm{~g} / \mathrm{j}$ ). Un accroissement de cette fraction azotée a aussi été mis en évidence par Focant et al (1990) après extrusion du pois (104 vs $68 \mathrm{~g} / \mathrm{j}$ ) tandis que, pour d'autres graines protéagineuses pauvres en amidon : soja, coton, lupin, ce flux demeure pratiquement constant, que le régime soit complémenté par la graine crue ou par la graine traitée (Stern et al, 1985; Pena et al, 1986; Benchaar et al, 1991). Dans nos conditions expérimentales, le flux duodénal de $\mathrm{Nt}$ est toujours plus important que Nt ingéré, soit : $+5,8 \%$ pour la ration féverole crue et $+21,1 \%$ pour la ration féverole extrudée. Un gain azoté, dû au recyclage de l'urée apparaît pour des régimes à faible(s) teneur(s) en MA et/ou $N$ fermentescible; des hausses allant de 15 à $43 \%$ ont été très souvent rapportées dans la littérature (Loerch et al, 1983; Santos et al, 1984; Stern et al, 1985; Garrett et al, 1987; Benchaar et al, 1991). Pour la féverole extrudée, une baisse de la digestion des MA dans le rumen, entraîne une hausse du flux duodénal $\mathrm{N}$ alimentaire* confirmant ainsi l'effet protecteur de l'extrusion à $195{ }^{\circ} \mathrm{C}$ que nous avons mis en évidence in situ, sur vaches taries (Benchaar et al, 1992), à savoir : une réduction, de l'ordre de $9 \%$, de la dégradation ruminale des MA et une augmentation, de $32 \%$ environ, de la digestion apparente dans les régions postruminales, ces 2 valeurs étant très proches de celles qui sont obtenues in vivo. Une élévation du flux $N$ alimentaire à l'entrée de l'intestin grêle est également rapportée après l'extrusion du pois : $+19 \%$ (Focant et al, 1990) et des graines de soja $\left(149^{\circ} \mathrm{C}\right):+104 \%$ (Stern et al, 1985), de

* $\mathrm{N}$ alimentaire $+\mathrm{N}$ endogène 
coton $\left(150^{\circ} \mathrm{C}\right):+29 \%$ (Pena et al, 1986) et de lupin $\left(195^{\circ} \mathrm{C}\right):+72 \%$ (Benchaar et al, 1991). Par contre, McMeniman et Armstrong (1979) ont signalé que les flux duodénaux : Nt, $\mathrm{N}$ alimentaire et $\mathrm{N}$ bactérien ne sont pas modifiés après traitement thermique de la féverole; toutefois, la température d'extrusion utilisée dans leur expérimentation était certainement trop faible $\left(105^{\circ} \mathrm{C}\right)$ pour, d'une part, protéger efficacement les protéines alimentaires de la digestion ruminale et, d'autre part, augmenter l'amylolyse. La digestion intestinale apparente de $\mathrm{N}$ non ammoniacal est accrue lorsque la ration est supplémentée par la graine extrudée à la place de la graine crue $(76,9$ vs $64,3 \%$ ingéré). Dans des conditions expérimentales similaires, mais où les animaux recevaient une ration complémentée par de la graine de lupin, crue ou extrudée à $195^{\circ} \mathrm{C}$, les valeurs observées pour $\mathrm{N}$ non ammoniacal étaient respectivement de 50,9 et $72,4 \%$ ingéré; l'augmentation étant uniquement le fait de $\mathrm{N}$ alimentaire (Benchaar et al, 1991).

À partir des résultats obtenus, nous avons évalué les teneurs PDI pour les graines crues et extrudées. Le flux $\mathrm{Nt}$ duodénal étant supérieur à $\mathrm{Nt}$ ingéré, nous avons formulé l'hypothèse d'un déficit en $\mathrm{N}$ soluble; les tables de la valeur nutritive des aliments (Andrieu et al, 1989) nous ont fourni les PDI pour l'ensilage de maïs, le foin de ray-grass et le maïs grain; aliments qui entrent dans la composition du régime. Dans ces conditions, les teneurs PDIA, PDIN et PDIE ( $\mathrm{g} / \mathrm{kg}$ de MS) sont respectivement : 33,175 et 127 pour la graine crue et 58, 147 et 178 pour la graine extrudée. Par ailleurs, il convient de signaler que les valeurs PDIA, PDIN et PDIE estimées à partir de l'étude in situ (Benchaar et al, 1992) sont : 61,161 et 134 pour la féverole crue, et $79,171,152$ pour l'extrudée $\left(195^{\circ} \mathrm{C}\right)$.

De cette expérimentation, nous retiendrons que la substitution de la féverole crue par de la féverole extrudée à $195^{\circ} \mathrm{C}$ dans l'alimentation de vaches laitières en production entraîne une diminution de la digestion ruminale des MA alimentaires, d'une part, et une augmentation de l'amylolyse et de l'efficacité de la synthèse des protéines bactériennes, d'autre part. Par voie de conséquence, le flux duodénal et la digestion intestinale de $\mathrm{N}$ non ammoniacal sont accrus.

\section{RÉFÉRENCES}

Andrieu J, Demarquilly C, Sauvant D (1989) Tables of feeds used in France. In: Ruminant nutrition (Jarrige R, ed) INRA, Libbey J, Eurotext, Paris-Londres, 213-294

Benchaar C, Bayourthe C, Moncoulon R, Vernay $M$ (1991) Digestion ruminale et absorption intestinale des protéines du lupin extrudé chez la vache laitière. Reprod Nutr Dev 31, 655-665

Benchaar C, Cros P, Bayourthe C (1992) Effets de l'extrusion sur la dégradabilité ruminale et la digestibilité intestinale des protéines de la féverole et du lupin, in situ, chez la vache tarie. In : $y^{r e}$ Conf Eur Protéagineux. Angers 1-3 juin; 501-502

Binnerts WT, Van't Klooster AT, Frens AM (1968) Soluble chromium indicator measured by atomic absorption in digestion experiments. Vet Rec 82, 470

Cerning-Beroard J (1977) Effect of technological processing on the carbohydrate composition of horse beans and on the susceptibility of starch alpha-amylase. In: Protein quality from leguminous crops. Comm Eur Commun EUR 5686 EN, 125-135

Cerning-Beroard J, Filiatre A (1977) Characterization and distribution of soluble and insoluble carbohydrates in legume seeds: horse beans, peas, lupines. In: Protein quality from leguminous crops. Comm Eur Commun, EUR 5686 EN, 65-76

Cros $P$, Vernay M, Moncoulon $R$ (1991) In situ evaluation of the ruminal and intestinal degradability of extruded whole horsebeans. Reprod Nutr Dev 31, 249-255 
Ellis WC, Lascano C, Teeter T, Owens FN (1982) Solute and particulate flow markers. In: Protein Requirements for cattle, symposium (Owens FN, ed) Oklahoma State Univ Stillwater, 37-56

Faichney GJ (1980) Measurement in the sheep of the quantity and composition of rumen digesta and the fractional outflow of digesta constituents. Aust J Agric Res 31, 11291137

Focant M, Van Hoecke A, Vanbelle M (1990) The effect of two heat treatments (steam flaking and extrusion) on the digestion of Pisum sativum in the stomachs of heifers. Anim Feed Sci Technol 28, 303-313

Garrett JE, Goodrich RD, Meiske JC, Stern MD (1987) Influence of supplemental nitrogen source on digestion of nitrogen, dry matter and organic matter and on in vivo rate of ruminal protein degradation. J Anim Sci 64, 1801-1812

Jouany JP (1982) Volatile fatty acid and alcohol determination, in digestive contents, silage juices, bacterial cultures and anaerobic fermentor contents. Sci Alim 2, 131-144

Jouany JP, Thivend P (1972) Évolution postprandiale de la composition glucidique des corps microbiens du rumen en fonction de la nature des glucides du régime. I. Les protozoaires. Ann Biol Anim Biochim Biophys 12, 673-677

Journal Officiel des Communautés Européennes (1971) Méthodes d'analyse des aliments pour les animaux (20 Déc)

Loerch SC, Berger LL, Plegge SD, Fahey GC Jr (1983) Digestibility and rumen escape of soybean meal, blood meal, meat and bone meal and dehydrated alfalfa nitrogen. $J$ Anim Sci 57, 1037-1047

Madsen J, Hvelplund T (1988) The influence of different protein supply and feeding level on $\mathrm{pH}$, ammonia concentration and microbial protein synthesis in the rumen of cows. Acta Agric Scand 38, 115-125

McMeniman NP, Armstrong DG (1979) The flows of amino acids into the small intestine of cattle when fed heated and unheated beans (Vicia faba). J Agric Sci (Camb) 93, 181-188

Michalet-Doreau B, Bogaert C, Bauchart D (1985) Valeur nutritive des graines de soja crues ou extrudées pour les ruminants. Bull Tech CRZV Theix, INRA 59, 29-38

Michalet-Doreau B, Doreau $M$, Voisin A, Bogaert $C$ (1987) Effets de l'extrusion sur la valeur azotée des aliments pour ruminants. In: Cuisson-extrusion. Nantes 16-17 Sept, INRA, Paris (Les Colloques de I'INRA $n^{\circ} 41$ ), 249258

Owens FN, Zinn RA, Kim YK (1986) Limits to starch digestion in the ruminant small intestine. J Anim Sci63, 1634-1648

Pena F, Tagari H, Satter LD (1986) The effect of heat treatment of whole cottonseed on site and extent of protein digestion in dairy cows. JAnim Sci 62, 1423-1433

Santos KA, Stern MD, Satter LD (1984) Protein degradation in the rumen and amino acid absorption in the small intestine of lactating dairy cattle fed various protein sources. J Anim Sci 58, 244-255

Siddons RC, Paradine J, Beever DE, Cornell PR (1985) Ytterbium acetate as a particulatephase digesta flow marker. Br J Nutr 54, 509517

Stern MD, Santos KA, Satter LD (1985) Protein degradation in rumen and amino acid absorption in small intestine of lactating dairy cattle fed heat-treated soybeans. J Dairy Sci 68, 45-56

Tagari H, Pena F, Satter LD (1986) Protein degradation by rumen microbes of heat-treated whole cottonseed. J Anim Sci 62, 1732-1736

Theurer CB (1986) Grain processing effects on starch utilization by ruminants. J Anim Sci 63, 1649-1662

Thomas E, Trenkle A, Burrougs W (1979) Evaluation of protective agents applied to soybean meal fed to cattle. I. Laboratory measurements. J Anim Sci 49, 1337-1345

Ushida K, Lassalas B, Jouany JP (1985) Determination of assay parameters for RNA analysis in bacterial and duodenal samples by spectrophotometry. Influence of sample treatment and preservation. Reprod Nutr Dev 25 , 1037-1046

Van Dijk HJ, O'Dell GD, Perry PR, Grimes LW (1983) Extruded versus raw ground soybeans for dairy cows in early lactation. J Dairy Sci 66, 2521-2525

Van Soest PJ (1963) Use of detergents in the analysis of fibrous feeds. II. A rapid method 
for the determination of fiber and lignin. $J$ Assoc Off Agric Chem 46, 829-835

Van Soest PJ, Wine RH (1967) Use of detergents in the analysis of fibrous feeds IV. Determination of plant cell wall constituents. $J$ Assoc Off Agric Chem 50, 50-55

Van Soest PJ, Wine RH (1968) Determination of lignin and cellulose in acid detergent fiber permanganate. Assoc Off Anal Chem 51, 780-785
Verdouw H, Van Echteld CJA, Dekkers EMJ (1977) Ammonia determination based on indophenol formation within sodium salicylate. Water Res 12, 399-402

Vérité R, Demarquilly C (1978) Qualité des matières azotées des aliments pour ruminants. In: La vache laitière. INRA, Versailles, 143-158

Waldo DR (1973) Extent and partition of cereal grain starch digestion in ruminants. $J$ Anim Sci 37, 1062-1074 